\title{
EFFECTS OF NITROGEN AND PLANT DENSITY ON DWARF SUNFLOWER HYBRIDS
}

\author{
Süzer, S. \\ Trakya Agricultural Research Institute, Agronomy Department, \\ Hacilar Ezani Mevki, E-5 Karayolu, 22100, Edirne, Turkey
}

Received: April 10, 2010

Accepted: August 10, 2010

\section{SUMMARY}

This research was carried out to determine the seed yield and some yield components of two dwarf hybrids as compared to one standard-height sunflower hybrid (Helianthus annuus L.) at different nitrogen rates and planting densities. The study was carried out under natural rainfed conditions at the Thrace Agricultural Research Institute in Edirne-Turkey between 1999 and 2001. The experiments were set up in split-split plots in a randomized complete block design with three replications. The main plot treatments were three different-height sunflower hybrids, DW-1, DW-2, and Trakya-80. The sub plots were three levels of nitrogen, 0,60 , and $120 \mathrm{~kg} \mathrm{~N} / \mathrm{ha}$. The sub-sub plots were three planting densities, $10 \times 70$ (142,850 plants/ha), $15 \times 70$ (95,230 plants/ ha), and $20 \times 70 \mathrm{~cm}(71,430$ plants/ha). Based on marginal economic analyses, the economically optimal seed yield per hectare was obtained at $50 \mathrm{~kg} \mathrm{~N} /$ ha for DW-1 and at $80 \mathrm{~kg} \mathrm{~N} /$ ha for DW-2 and Trakya-80. In all three hybrids, increasing plant densities decreased 1000-seed weight, hull percentage, and head diameter but increased test weight in natural rainfed conditions. The highest seed yield in both dwarf hybrids was obtained with the spacing of $15 \times 70 \mathrm{~cm}(95,230$ plants/ha). The results of this research show that nitrogen and plant density have significant effects on seed yield and some yield components of different-height sunflower hybrids.

Key words: sunflower, dwarf, nitrogen, plant density

\section{INTRODUCTION}

The sunflower (Helianthus annuus L.) is one of the most widely cultivated oil crops in the world. It is also grown widely in the Trakya-Marmara region of Turkey. Turkish farmers use oilseed sunflower varieties of different heights. These different varieties needs different plant densities and nitrogen requirements in order to produce high seed and oil yields per hectare. In particular, early-maturing dwarf and semi-dwarf hybrids used for commercial production have led to a change in plant

* Corresponding author: Phone: +90-(284)-235 81 82-404;

e-mail: suzersami@yahoo.com 
population, row spacing and fertilizer rate recommendations compared to those used for conventional hybrids. Dwarf hybrids generally perform the best when grown at high plant populations, and they also maintain yield levels better than conventional hybrids when planted on later dates (Majid and Schneiter, 1987; Gubbels and Dedio, 1990; Hussain, 1990; Feoli and Schneiter, 1993; Süzer and Atakişi, 1994; Göksoy and Turan, 1999). In commercial sunflower production, higher plant populations are recommended for dwarf sunflower hybrids when the grower wants to achieve optimum seed yield performance.

Some advantages of the reduced-height hybrids are high resistance to lodging and easy cultivation and harvesting. Scientific data on the performance and agronomic characteristics of the reduced-height hybrids at different plant populations per hectare is limited. Stand density can influence sunflower grain yields, which depend on three components. These are the number of heads per hectare, number of seeds per head, and 1000-seed weight. The optimum plant population used in various production areas throughout the world shows variation. In some experiments, the results showed that higher seed yields and oil contents were obtained with increasing plant populations (Robinson et al., 1980; Vannozzi and Baldini, 1988). However, it has also been reported that different plant populations had no effect on seed yield (Prunty, 1983).

Plant population affects other plant characteristics as well. As plant population is increased from low to high, the flowering stage was delayed, plant height increased, the plants lodged more, and seed size, head and stalk diameter decreased (Vranceanu et al., 1982; Brigham and Young, 1985; Fick et al., 1985; Miller and Hommond, 1989). The performances of tall, semi-dwarf and dwarf sunflower hybrids are different at various plant populations. The reduced-height sunflower hybrids responded better to high plant populations than the standard and tall hybrids did (Stanojević, 1989; Vannozi and Baldini, 1990; Feoli, 1993; Fick et al., 1985; Johnson, 2002).

Many fertilizer studies have shown that sunflower responds to fertilizer use. Nitrogen, phosphorus and potassium (NPK) in particular are few of the major nutrients required to significantly increase sunflower yields. Fertilizer application rates in sunflower production vary because of the variable occurrence of NPK in the soil. Nitrogen is a common element limiting sunflower yields (Süzer and Uludere, 1997; Süzer, 1998).

In the Trakya region of Turkey, some farmers believe that oilseed sunflowers do not require as much fertilizer as the other field crops. Actually, sunflower has an extensive root system that may help in the utilization of residual soil nutrients that have been given to wheat as a rotation crop. According to researches on sunflower production, the $\mathrm{N}$ budget/ha in the soil for a $2,000 \mathrm{~kg}$ seed crop amounts to $48 \mathrm{~kg}$ for the seed, $31 \mathrm{~kg}$ for the stover, $3 \mathrm{~kg}$ for the roots and $14 \mathrm{~kg}$ for the soil microflora - a grand total of about $100 \mathrm{~kg} \mathrm{~N}$ (Robinson, 1978). 
The objectives of this study were to determine the seed yield and some yield components of two dwarf hybrids compared with one standard-height sunflower hybrid at different nitrogen rates and planting densities under natural rainfed conditions.

\section{MATERIALS AND METHODS}

This research was conducted between 1999 and 2001 at the Thrace Agricultural Research Institute in Edirne, which is located in the European part of Turkey. The main properties of the soil used in the field experiments are presented in Table 1. The fertilizer used in the experiment area was in accordance with the results of soil analyses.

Table 1: Main properties of soil used in the field experiments.

\begin{tabular}{lcccccccccc}
\hline Year & $\begin{array}{c}\text { Depth } \\
(\mathrm{cm})\end{array}$ & $\begin{array}{c}\text { Dry/ } \\
\text { wet }\end{array}$ & $\begin{array}{c}\text { Water satu- } \\
\text { ration (\%) }\end{array}$ & $\mathrm{pH}$ & $\begin{array}{c}\text { Texture } \\
\text { class }\end{array}$ & $\begin{array}{c}\mathrm{P}_{2} \mathrm{O}_{5} \\
(\mathrm{ppm})\end{array}$ & $\begin{array}{c}\mathrm{K}_{2} \mathrm{O} \\
(\mathrm{ppm})\end{array}$ & $\begin{array}{c}\mathrm{Ca} \\
(\mathrm{ppm})\end{array}$ & $\begin{array}{c}\mathrm{Zn} \\
(\mathrm{ppm})\end{array}$ & $\begin{array}{c}\text { Organic } \\
\text { matter }(\%)\end{array}$ \\
\hline 1999 & $0-30$ & Dry & 50 & 6.07 & Silt & 33.4 & 351.0 & 2442 & 0.867 & 1.4 \\
2000 & $0-30$ & Dry & 50 & 6.12 & Silt & 26.7 & 316.0 & 2665 & 0.459 & 1.1 \\
2001 & $0-30$ & Dry & 44 & 6.26 & Silt & 32.7 & 337.0 & 2612 & 0.659 & 1.2 \\
\hline
\end{tabular}

Three oilseed-type sunflower hybrids of different height (two dwarf and one standard), DW-1, DW-2, and Trakya-80, were used as the experiment material. The hybrids were developed at the Trakya Agricultural Research Institute in Edirne.

The experiments were established in a split-split plot design in RCBD with three replications. The main plots had sunflower hybrids of three different heights: DW-1, DW-2, and Trakya-80. The sub plots were three different nitrogen rates; 0 , 60 , and $120 \mathrm{~kg} \mathrm{~N}$. The sub-sub plots were three different planting densities; $10 \times$ $70 \mathrm{~cm}(142,850$ plants/ha), $15 \times 70 \mathrm{~cm}(95,230$ plants/ha $)$ and $20 \times 70 \mathrm{~cm}(71,430$ plants/ha).

Plot size at planting was $2.8 \times 7.5 \mathrm{~m}=21.0 \mathrm{~m}^{2}$, while plot size at harvesting was $1.4 \times 6.9=9.7 \mathrm{~m}^{2}$. The fertilizer required for each plot according to the experimental design was broadcast prior to planting manually. The intra-row spacing was 10 , 15 , and $20 \mathrm{~cm}$, while the rows were spaced $70 \mathrm{~cm}$ apart.

The trial was carried out in rotation with wheat. The materials were planted in April. The hills on the plots were over-planted and then thinned to one plant per hill three weeks after sowing. Weed control was accomplished using both chemicals and cultural practices.

Mean values of seed yield, oil content, oil yield, 1000-seed weight, test weight, plant height, head diameter, time to flowering and time to physiological maturity were determined in each plot and analyzed using ANOVA. Regression analysis was applied to find the relationships between fertilizer rates and seed yield and also between fertilizer rates and oil yield (Little and Hills, 1978; Russell, 1986). 


\section{RESULTS AND DISCUSSION}

Presented in Tables 2, 3, 4, and 5 are the effects of different nitrogen rates and planting densities on seed yield and some yield components of dwarf and standard height sunflower hybrids under natural rainfed conditions between 1999 and 2001. The relationship between the seed yields of DW-1, DW-2 and Trakya-80 and nitrogen rates is shown in Figure 1. The maximum and economically optimal seed yields of the three sunflower hybrids were calculated from regression equations as a response to fertilizer rates and are given in Table 6.

As seen in Table 2, as a main plot, the two dwarf and one standard-height sunflower hybrids affected significantly $(\mathrm{p} \leq 0.01$ and $\mathrm{p} \leq 0.05)$ the mean oil content, oil yield, 1000-seed weight, test weight, hull percentage, and plant height at three nitrogen rates and planting densities over three study years. In the three-year growing period, standard-height sunflowers outperformed the two dwarf hybrids and gave the highest seed yield $(2,266 \mathrm{~kg} / \mathrm{ha})$. Feoli et al. (1993) obtained similar results when comparing the agronomic performance of dwarf, semi-dwarf, and conventional-height sunflower hybrids grown at five plant populations under rainfed field conditions in North Dakota, USA.

Table 2: As a main plot, mean seed yield and yield components of two dwarf and one standard-height sunflower hybrids at three nitrogen rates and planting densities between 1999 and 2001.

\begin{tabular}{lcccccccccc}
\hline \multirow{2}{*}{ No. } & Variety & $\begin{array}{c}\text { Seed } \\
\text { yield }\end{array}$ & $\begin{array}{c}\text { Yield } \\
\text { rank }\end{array}$ & $\begin{array}{c}\text { Oil in } \\
\text { seed }\end{array}$ & $\begin{array}{c}\text { Oil } \\
\text { yield }\end{array}$ & $\begin{array}{c}\text { 1000-seed } \\
\text { weight }\end{array}$ & $\begin{array}{c}\text { Test } \\
\text { weight }\end{array}$ & Hull & $\begin{array}{c}\text { Plant } \\
\text { height }\end{array}$ & $\begin{array}{c}\text { Head } \\
\text { diameter }\end{array}$ \\
\cline { 2 - 11 } & & $(\mathrm{kg} / \mathrm{ha})$ & & $(\%)$ & $(\mathrm{kg} / \mathrm{ha})$ & $(\mathrm{g})$ & $(\mathrm{kg} / \mathrm{HI})$ & $\%$ & $(\mathrm{~cm})$ & $(\mathrm{cm})$ \\
\hline 1 & DW-1 & 1988 & 2 & 38.4 & 763 & 41.8 & 40.8 & 28.3 & 84.7 & 11.6 \\
2 & DW-2 & 1934 & 3 & 39.2 & 759 & 40.1 & 42.5 & 27.8 & 82.2 & 11.6 \\
3 & Trakya-80 & 2266 & 1 & 43.6 & 983 & 36.2 & 40.3 & 24.4 & 122.0 & 12.4 \\
\hline LSD (0.05) & $473^{\text {ns }}$ & & $0.5^{\star \star}$ & $150^{\star}$ & $1.7^{\star}$ & $1.6^{\star}$ & $0.8^{\star}$ & $15.2^{\star \star}$ & 0.3 \\
C.V.(\%) & 12.1 & & 2.3 & 9.1 & 4.9 & 1.0 & 4.4 & 5.1 & 7.4 \\
\hline
\end{tabular}

${ }^{*},{ }^{* *}$ : Significant at 0.05 and 0.01 levels.

Table 3: Mean seed yield and yield components of sunflowers as affected by three levels of nitrogen as a sub plot average of three seasons (1999 to 2001).

\begin{tabular}{lcccccccccc}
\hline \multirow{2}{*}{ No. } & $\begin{array}{c}\text { Nitrogen } \\
\text { rates }\end{array}$ & $\begin{array}{c}\text { Seed } \\
\text { yield }\end{array}$ & $\begin{array}{c}\text { Yield } \\
\text { rank }\end{array}$ & $\begin{array}{c}\text { Oil in } \\
\text { seed }\end{array}$ & $\begin{array}{c}\text { Oil } \\
\text { yield }\end{array}$ & $\begin{array}{c}\text { 1000-seed } \\
\text { weight }\end{array}$ & $\begin{array}{c}\text { Test } \\
\text { weight }\end{array}$ & Hull & $\begin{array}{c}\text { Plant } \\
\text { height }\end{array}$ & $\begin{array}{c}\text { Head } \\
\text { diameter }\end{array}$ \\
\cline { 2 - 11 } & (ha) & $(\mathrm{kg} / \mathrm{ha})$ & & $(\%)$ & $(\mathrm{kg} / \mathrm{ha})$ & $(\mathrm{g})$ & $(\mathrm{kg} / \mathrm{Hl})$ & $(\%)$ & $(\mathrm{cm})$ & $(\mathrm{cm})$ \\
\hline 1 & $\mathrm{~N} 0$ & 1853 & 3 & 39.8 & 739 & 39.4 & 41.6 & 26.5 & 95.7 & 11.3 \\
2 & $\mathrm{~N} 60$ & 2168 & 1 & 40.4 & 879 & 39.6 & 41.2 & 27.8 & 96.3 & 11.9 \\
3 & $\mathrm{~N} 120$ & 2166 & 2 & 40.4 & 876 & 39.1 & 41.0 & 26.6 & 97.0 & 12.4 \\
\hline $\mathrm{LSD}(0.05)$ & $246^{\star \star}$ & & $0.4^{\star \star}$ & $71^{\star \star}$ & 0.9 & $0.6^{\star \star}$ & $0.5^{\star *}$ & 3.9 & $0.6^{\star}$ \\
$\mathrm{C} . \mathrm{V} .(\%)$ & 12.1 & & 2.3 & 9.1 & 4.9 & 1.0 & 4.4 & 5.1 & 7.4 \\
\hline
\end{tabular}

${ }^{*},{ }^{* *}$ : Significant at 0.05 and 0.01 levels.

As seen in Table 3, as a sub plot, nitrogen rates affected the mean seed yield, oil content, oil yield, test weight, hull percentage, and head diameter of two dwarf and one standard-height sunflower hybrids significantly ( $\mathrm{p} \leq 0.01$ and $\mathrm{p} \leq 0.05$ ). Based on three-year growing season data, the nitrogen rate of $60 \mathrm{~kg} /$ ha gave the highest mean 
seed yield (2,168 kg/ha). Similar results have been reported by Arslan (1989) and Süzer (1998), who conducted studies on nitrogen rates in sunflower under Trakya field conditions.

As seen in Table 4, as a sub-sub plot, three plant densities affected the mean 1000-seed weight, test weight, hull percentage, and head diameter of two dwarf and one standard-height sunflower hybrids significantly ( $\mathrm{p} \leq 0.01$ and $\mathrm{p} \leq 0.05$ ). Based on three-year growing season data, the spacing of $15 \times 70 \mathrm{~cm}$, or 95,230 plants per hectare, gave the highest mean seed yield $(2,132 \mathrm{~kg} / \mathrm{ha})$ of all the plant densities.

Table 4: Mean seed yield and yield components of sunflower as affected by three planting densities as a sub-sub plot average of three seasons (1999 to 2001).

\begin{tabular}{lcccccccccc}
\hline \multirow{2}{*}{ No. $\begin{array}{c}\text { Plant } \\
\text { density }\end{array}$} & $\begin{array}{c}\text { Seed } \\
\text { yield }\end{array}$ & $\begin{array}{c}\text { Yield } \\
\text { rank }\end{array}$ & $\begin{array}{c}\text { Oil in } \\
\text { seed }\end{array}$ & $\begin{array}{c}\text { Oil } \\
\text { yield }\end{array}$ & $\begin{array}{c}\text { 1000-seed } \\
\text { weight }\end{array}$ & $\begin{array}{c}\text { Test } \\
\text { weight }\end{array}$ & Hull & $\begin{array}{c}\text { Plant } \\
\text { height }\end{array}$ & $\begin{array}{c}\text { Head } \\
\text { diameter }\end{array}$ \\
\hline & & $(\mathrm{kg} / \mathrm{ha})$ & & $(\%)$ & $(\mathrm{kg} / \mathrm{ha})$ & $(\mathrm{g})$ & $(\mathrm{kg} / \mathrm{Hl})$ & $\%$ & $(\mathrm{~cm})$ & $(\mathrm{cm})$ \\
\hline 1 & $10 \times 70$ & 2014 & 3 & 40.4 & 814 & 37.9 & 42.0 & 26.5 & 96.1 & 11.1 \\
2 & $15 \times 70$ & 2132 & 1 & 40.5 & 866 & 39.2 & 41.3 & 26.8 & 95.9 & 12.0 \\
3 & $20 \times 70$ & 2043 & 2 & 39.9 & 817 & 41.6 & 40.6 & 27.5 & 97.0 & 12.7 \\
\hline LSD(0.05) & 367 & & 0.8 & 10.1 & $2.4^{\star *}$ & $0.7^{\star *}$ & $1.2^{\star *}$ & 6.3 & $1.1^{\star *}$ \\
C.V.(\%) & 12.1 & & 2.3 & 9.1 & 4.9 & 1.0 & 4.4 & 5.1 & 7.4 \\
\hline
\end{tabular}

*, ** : Significant at 0.05 and 0.01 levels

As seen in Figure 1 and Table 6 , the correlation coefficient values of DW-1 $\left(\mathrm{R}=0.778^{* *}\right), \mathrm{DW}-2\left(\mathrm{R}=0.857^{* *}\right)$ and Trakya-80 $\left(\mathrm{R}=0.833^{* *}\right)$ were found to be significant. Regression equations for DW-1 $\left(\mathrm{Y}=1906.0+4.56 \mathrm{X}-0.031 \mathrm{X}^{2}\right)$, DW-2 $\left(\mathrm{Y}=1636.0+11.18 \mathrm{X}-0.064 \mathrm{X}^{2}\right)$ and Trakya-80 $\left(\mathrm{Y}=2019.6+8.35 \mathrm{X}-0.042 \mathrm{X}^{2}\right)$ were also found to be significant. Based on marginal economic analyses, the economically optimal seed yield per hectare was obtained at $50 \mathrm{~kg} \mathrm{~N} / \mathrm{ha}$ for DW-1 and at 80 $\mathrm{kg}$ N/ha for DW-2 and Trakya-80.

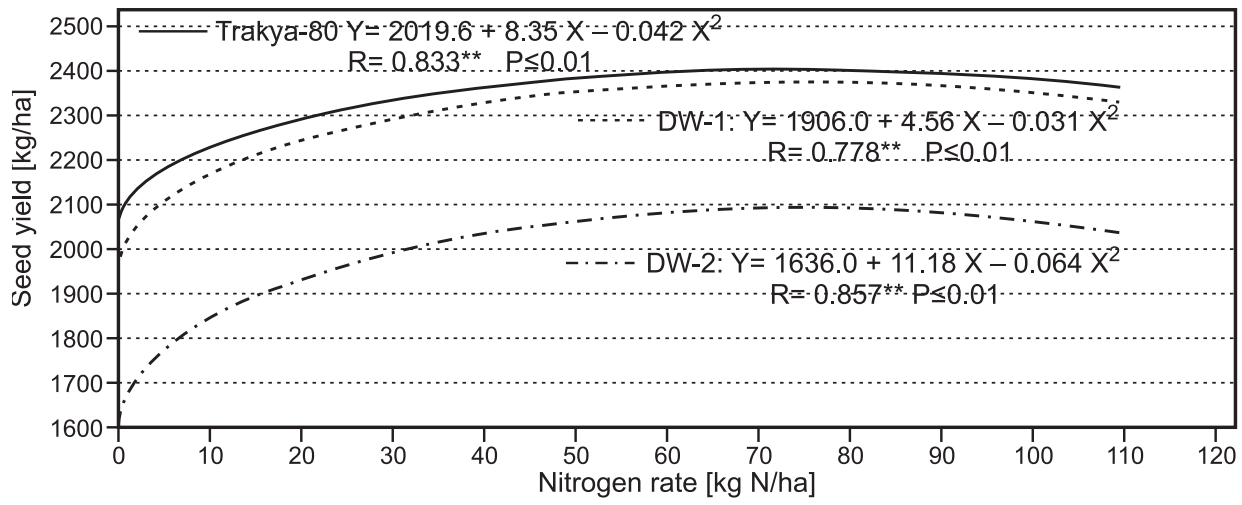

Figure 1: Relationship between seed yields of DW-1, DW-2 and Trakya-80 and nitrogen rates.

In all three hybrids, increasing plant densities decreased 1000-seed weight, hull percentage, and head diameter but increased test weight in natural rainfed conditions. The highest seed yield in both dwarf hybrids was obtained with the spacing of $15 \times 70 \mathrm{~cm}(95,230$ plants/ha). 
As seen in Table 5, the highest seed yield (2,585 kg/ha) was obtained at $120 \mathrm{~kg}$ $\mathrm{N} / \mathrm{ha}$ and the spacing of $20 \times 70 \mathrm{~cm}(71,430$ plants/ha) with the standard-height hybrid Trakya- 80 . The second highest seed yield $(2,231 \mathrm{~kg} / \mathrm{ha})$ was obtained at 120 $\mathrm{kg} \mathrm{N} / \mathrm{ha}$ and $15 \times 70 \mathrm{~cm}(95,230$ plants/ha) with DW-2. DW-1, however, produced the highest seed yield at $60 \mathrm{~kg} \mathrm{~N} / \mathrm{ha}$ and a plant density of $15 \times 70 \mathrm{~cm}(95,230$ plants/ha).

Table 5: Mean seed yield and yield components of three sunflower hybrids as affected by three nitrogen levels and three planting densities over three seasons (1999 to 2001).

\begin{tabular}{|c|c|c|c|c|c|c|c|c|c|c|c|c|c|c|}
\hline 윽 & 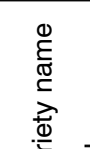 & 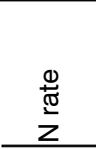 & 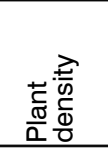 & $\begin{array}{l}\bar{\delta} \frac{0}{0} \\
\mathbb{D} \frac{0}{2} \\
\infty\end{array}$ & 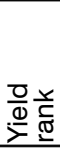 & 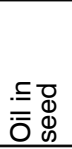 & $\begin{array}{l}\frac{0}{0} \\
\frac{0}{\overline{0}}\end{array}$ & 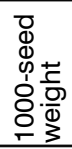 & 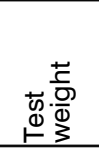 & $\begin{array}{l}\overline{\overline{3}} \\
\text { 工 }\end{array}$ & 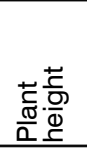 & 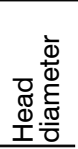 & 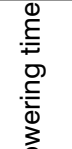 & 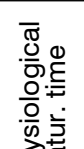 \\
\hline & & $\overline{(\mathrm{kg} / \mathrm{ha})}$ & & $(\mathrm{kg} / \mathrm{ha})$ & & $(\%)$ & $(\mathrm{kg} / \mathrm{ha})$ & (g) & $(\mathrm{kg} / \mathrm{HI})$ & $\%$ & $(\mathrm{~cm})$ & (cm) & 은 & \\
\hline 1 & & 0 & $0 \times 70$ & 1938 & 20 & 36.4 & 704 & 41.5 & 42.2 & 28.1 & 83.9 & 10.6 & 64.6 & 107.2 \\
\hline 0 & & 0 & $\times 70$ & 1899 & 22 & 37.1 & 705 & 42.5 & 41.3 & 28.7 & 84.3 & 11.1 & 64.3 & 108.7 \\
\hline 3 & & 0 & $20 \times 70$ & 1882 & 23 & 37.3 & 702 & 45.5 & 40.3 & 29.4 & 86.5 & 11.6 & 63.3 & 109.5 \\
\hline I & & 60 & $10 \times 70$ & 2033 & 15 & 38.3 & 779 & 39.8 & 41.5 & 29.1 & 82.8 & 10.4 & 61.7 & 107.2 \\
\hline 5 & WW 1 & 60 & $\times 70$ & 2097 & 11 & 37.6 & 789 & 41.3 & 40.2 & 29.2 & 82.8 & 11.3 & 62.5 & 107.3 \\
\hline 6 & & 60 & $20 \times 70$ & 2057 & 13 & 38.5 & 790 & 44.4 & 40.4 & 28.1 & 85.7 & 12.5 & 62.2 & 107.7 \\
\hline 7 & & 1 & $\times 70$ & 2036 & 14 & & 805 & 39.4 & 41.4 & 27.2 & 87.8 & 11.6 & 61.3 & 106.3 \\
\hline 8 & & 12 & $15 \times 70$ & 2070 & 12 & 39.5 & 816 & 39.9 & 40.9 & 28.7 & 83.2 & 12.0 & 61.2 & 107.0 \\
\hline 9 & & 18 & $\times 70$ & 1881 & 24 & 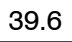 & 743 & 42.5 & 39.7 & 27.1 & 85.6 & 13.1 & 1.8 & 107.0 \\
\hline 10 & & 0 & $10 \times 70$ & 1579 & 26 & 37. & 591 & 36.5 & 44.4 & 28.1 & 77.9 & 9.4 & 66.0 & 111.5 \\
\hline & & $c$ & $\times 70$ & 761 & 25 & 39. & 696 & 40.5 & 43.3 & 26.3 & 80.8 & 11.1 & 5.7 & 111.5 \\
\hline 12 & & 0 & $20 \times 70$ & 1568 & 27 & 38.4 & 601 & 43.4 & 41.8 & 27.1 & 84.8 & 12.1 & 65.3 & 111.8 \\
\hline 13 & & 6 & $\times 70$ & 2117 & 9 & 40.3 & 856 & 38.1 & 43.5 & 28.5 & 80.5 & 11.0 & 64.0 & 110.7 \\
\hline 14 & W/ & 6 & 0 & 2207 & 8 & 38. & 870 & 41.5 & 42.9 & 27.5 & 83.7 & 12.3 & 65.0 & 111.2 \\
\hline 15 & & 60 & $\times 70$ & 1901 & 21 & 39. & 748 & 41.8 & 41.5 & 28.5 & 83.3 & 12.6 & 65.2 & 111.2 \\
\hline 1 & & & 0 & 2027 & 16 & 40 & 822 & 37.4 & 42.8 & 28.1 & 82.0 & 11.5 & 63.8 & 107.8 \\
\hline 17 & & 120 & $\times 70$ & 2231 & 5 & 40.3 & 899 & 40.3 & 42.2 & 27.5 & 84.7 & 12.6 & 63.7 & 108.2 \\
\hline $1 \varepsilon$ & & & $\times 70$ & 2021 & 17 & 38.4 & 776 & 41.1 & 41.0 & 29.1 & 82.3 & 12.0 & 63.5 & 108.7 \\
\hline 19 & & 0 & $\times 70$ & 1965 & 19 & 3 & 856 & 30.6 & 40.8 & 24.4 & 122.8 & 11.3 & 66.0 & 112.3 \\
\hline 2 & & 0 & $\times 70$ & 1990 & 18 & 44 & 892 & 34.3 & 39.9 & 23.5 & 119.6 & 12.1 & 67.2 & 113.7 \\
\hline 21 & & 0 & $20 \times 70$ & 2104 & 10 & 43. & 912 & 40.6 & 40.2 & 23.3 & 121.0 & 12.6 & 67.0 & 112.3 \\
\hline $2<$ & & 00 & $10 \times 70$ & 2215 & 7 & 43.8 & 970 & 34.7 & 40.6 & 24.5 & 125.8 & 11.3 & 64.7 & 109.8 \\
\hline 23 & P & 60 & 0 & 2497 & 2 & 44.1 & 102 & 34.6 & 39.9 & 25.2 & 120.1 & 12.1 & 64.0 & 110.0 \\
\hline 24 & & 60 & $20 \times 70$ & 2393 & 4 & 43.6 & 1044 & 40.2 & 40.3 & 28.6 & 121.6 & 13.4 & 64.2 & 110.7 \\
\hline 25 & & 120 & $\times 70$ & 2213 & 6 & 43. & 967 & 37.8 & 40.9 & 23.3 & 121.3 & 12.1 & 64.5 & 110.2 \\
\hline 26 & & 12 & & 2437 & 3 & 42.6 & 1037 & 37.9 & 40.4 & 23.7 & 123.7 & 12.9 & 64.2 & 110.2 \\
\hline 27 & & 11 & $20 \times 70$ & 2585 & 1 & 40.6 & 1049 & 35.3 & 39.9 & 24.5 & 122.2 & 13.9 & 64.0 & 110.5 \\
\hline \multicolumn{3}{|c|}{$\overline{L S D}(0.05)$} & & $425^{*}$ & & 1 & $113^{\star \star}$ & $3.2^{\star \star}$ & $0.8^{\star \star}$ & $2.1^{\star \star}$ & $7.9^{\star \star}$ & $0.9^{\star \star}$ & $1.5^{\star \star}$ & $1.0^{* \star}$ \\
\hline \multicolumn{3}{|c|}{ C.V. (\%) } & & 12.1 & & 2.3 & 9.1 & 4.9 & 1.3 & 4.4 & 5.1 & 7.4 & 0.8 & 0.6 \\
\hline
\end{tabular}

*, **: Significant at 0.05 and 0.01 levels. 
Table 6: Maximum and economically optimal seed yields of three sunflower hybrids as calculated from regression equations as a response to fertilizer rates.

\begin{tabular}{|c|c|c|c|c|c|c|c|c|c|}
\hline \multirow{2}{*}{$\begin{array}{l}\text { Nitrogen } \\
\text { rate }\end{array}$} & \multicolumn{3}{|c|}{$\begin{array}{c}\text { DW-1 } \\
Y=1906.0+4.56 X-0.031 X^{2} \\
\left(R=0.778^{\star \star}\right)\end{array}$} & \multicolumn{3}{|c|}{$\begin{array}{c}\text { DW-2 } \\
Y=1636.0+11.18 X-0.064 X 2 \\
\left(R=0.857^{\star \star}\right)\end{array}$} & \multicolumn{3}{|c|}{$\begin{array}{c}\text { Trakya-80 } \\
\mathrm{Y}=2019.6+8.35 \mathrm{X}-0.042 \mathrm{X}^{2} \\
\left(\mathrm{R}=0.833^{\star \star}\right)\end{array}$} \\
\hline & $\begin{array}{l}\text { Total } \\
\text { yield }\end{array}$ & $\begin{array}{c}\text { Marginal } \\
\text { yield }\end{array}$ & $\begin{array}{l}\text { Value of } \\
\text { marginal } \\
\text { yield }\end{array}$ & $\begin{array}{l}\text { Total } \\
\text { yield }\end{array}$ & $\begin{array}{c}\text { Marginal } \\
\text { yield }\end{array}$ & $\begin{array}{l}\text { Value of } \\
\text { marginal } \\
\text { yield }\end{array}$ & $\begin{array}{l}\text { Total } \\
\text { yield }\end{array}$ & $\begin{array}{c}\text { Marginal } \\
\text { yield }\end{array}$ & $\begin{array}{l}\text { Value of } \\
\text { marginal } \\
\text { yield }\end{array}$ \\
\hline (kg/ha) & (kg/ha) & $(\mathrm{kg} / \mathrm{da})$ & (TL/ha) & (kg/ha) & (kg/da) & (TL/ha) & (kg/ha) & (kg/da) & (TL/ha) \\
\hline 0 & 1906.0 & 0.0 & 0.00 & 1636.0 & 0.0 & 0.00 & 2019.6 & 0.0 & 0.00 \\
\hline 10 & 1948.5 & 42.5 & 25.08 & 1741.4 & 105.4 & 62.18 & 2098.9 & 79.3 & 46.78 \\
\hline 20 & 1984.8 & 36.3 & 21.42 & 1834.0 & 92.6 & 54.63 & 2232.3 & 70.9 & 41.83 \\
\hline 30 & 2014.9 & 30.1 & 17.76 & 1913.8 & 79.8 & 47.08 & 2286.4 & 62.5 & 36.87 \\
\hline 40 & 2038.8 & 23.9 & 14.10 & 1980.8 & 67.0 & 39.53 & 2332.1 & 54.1 & 31.91 \\
\hline 50 & 2056.5 & 17.7 & 10.44 & 2035.0 & 54.2 & 31.97 & 2362.1 & 45.7 & 26.96 \\
\hline 60 & 2068.0 & 11.5 & 6.78 & 2076.4 & 41.4 & 24.42 & 2369.4 & 37.3 & 22.00 \\
\hline 70 & 2073.3 & 5.3 & 3.12 & 2105.0 & 28.6 & 16.87 & 2398.3 & 28.9 & 17.05 \\
\hline 80 & 2072.4 & -0.9 & -0.53 & 2120.8 & 15.8 & 9.32 & 2418.8 & 20.5 & 12.09 \\
\hline 90 & 2065.3 & -7.1 & -4.18 & 2123.8 & 3.0 & 1.77 & 2430.9 & 12.1 & 7.13 \\
\hline 100 & 2052.0 & -13.3 & -7.85 & 2114.0 & -9.8 & -5.78 & 2434.6 & 3.7 & 2.18 \\
\hline 110 & 2032.5 & -19.5 & -11.50 & 2091.4 & -22.6 & -13.33 & 2429.9 & -4.7 & -2.77 \\
\hline 120 & 2006.8 & -25.7 & -15.16 & 2056.0 & -35.4 & -20.88 & 2416.8 & -13.1 & -7.72 . \\
\hline
\end{tabular}

*: $1 \mathrm{~kg}$ ammonium nitrate fertilizer price in 2001 in Turkey (\%26 N): $0.22 \mathrm{TL}$

**: $1 \mathrm{~kg}$ oilseed sunflower price in 2001 in Turkey: $0.59 \mathrm{TL}$

\section{CONCLUSIONS}

Nitrogen fertilizer rates increased seed yield, oil content, oil yield, hull percentage and head diameter of three different-height sunflower hybrids in natural rainfed conditions significantly $(\mathrm{p} \leq 0.01$ and $\mathrm{p} \leq 0.05)$. Regression equations for $\mathrm{DW}-1$ $\left(\mathrm{Y}=1906.0+4.56 \mathrm{X}-0.031 \mathrm{X}^{2}\right), \mathrm{DW}-2\left(\mathrm{Y}=1636.0+11.18 \mathrm{X}-0.064 \mathrm{X}^{2}\right)$ and Trakya$80\left(Y=2019.6+8.35 \mathrm{X}-0.042 \mathrm{X}^{2}\right)$ were found to be significant. Based on marginal economic analyses, the economically optimal seed yield per hectare was obtained at $50 \mathrm{~kg} \mathrm{~N} / \mathrm{ha}$ for DW-1 and at $80 \mathrm{~kg} \mathrm{~N} / \mathrm{ha}$ for DW-2 and Trakya-80.

In all three hybrids, increasing plant densities decreased 1000-seed weight, hull percentage, and head diameter but increased test weight in natural rainfed conditions.

As a result, the highest seed and oil yields under the rainfed conditions of Edirne in Turkey were produced by the two dwarf hybrids at $15 \times 70 \mathrm{~cm}(95,230$ plant/ha). 


\section{REFERENCES}

Arslan, R., 1989. Trakya koşullarinda azotlu, fosforlu gübre istegi ve olsen fosfor analiz metodunun kalibrasyonu. Kirklareli Atatürk Araştirma Enst. Müdürlügü. Genel Yayin No: 15. Rapor Serisi No: 11, sayfa: 86.

Johnson, B.L., 2002. Dwarf sunflower response to row spacing, stand reduction, and defoliation at different growth stages. Canadian Journal of Plant Science 83: 319-326.

Feoli, C.E., Schneiter, A.A. and Johnson, B.L., 1993. Agronomic performance of dwarf, semidwarf, and conventional height sunflower hybrids grown at five plant populations under rainfed codititions. Helia 16(19):19-30.

Fick, G.N. Caroline, J.J., Auvartir, G.E. ve Duhing, P.M., 1985. Agronomic characteristics and field performance of dwarf sunflower hybrids. In: XI Intl. Sunf. Conference, 10-13 March 1985. Mardelplata, Argentina. S: 379-742.

Goksoy, A.T. and Turan, Z.M., 1999. Effects of stand reduction applied at different plant growth stages on the yield and yield components of sunflowers. Turk. J. Agric. For. 23: 329-335.

Majid, H.R., and Schneiter, A.A., 1987. Yield and quality of semidwarf and standard height sunflower hybrids grown at five populations. Agron. Journal, 79:681-684.

Robinson, R.G., Ford, J.H., Lueschen Kabas, D.L., Smith, L.J., Warnes, D.D. and Wiersma, J.V., 1980. Response of sunflower to plant population. Agron. J. 72: 869-871.

Russell, F. 1986. Microcomputer statistical program (MSTAT) Version 4.00/EM. Mcihigan State University. Mstat/crop and soil sciences. 324B. Agricultural Hall. East lansing, Michigan. USA.

Schneiter, A.A. and Miller, J.F., 1981. Description of Sunflower Growth Stages. Crop Sci. 21: 901-903.

Süzer, S. and Atakişi, I., 1993. Yield components of sunflower hybrids of different height. Helia 16(18): 35-40.

Süzer, S., and Uludere, A.Ö., 1997. Effects of nitrogen, phosphorus and potassium on yield and yield components of sunflower under irrigated conditions. The International Potash Institute's Regional Workshop on Food Security in WANA Region, The essential need for balanced fertilizitation. May 26-30, 1997. Bornova, Turkey, pp. 191-202.

Süzer, S., 1998. Effect Of Different Forms Of Nitrogen Fertilizers, Rates And Application Times On Sunflower Yield And Yield Components. Proceeding of $2^{\text {nd }}$ Balkan Symposium on Field Crops. Novi Sad, Yugoslavia. 16-20 June. 1998, 2: 219-223.

Vannozzi, G.P., and Baldini, M., 1988. The effect of plant density and "rectangularity" on achnene yield in long and short stemmed sunflower cultivars. XII International Sunflower Conf., Yugoslavia, 1: 392-394.

Vranceanu, A.V., Stoenescu, F.M. and Terbea, M., 1982. Tolerance of sunflower hybrids to competion among plants. Helia 5: 23-26.

Yurtsever, N., 1984. Deneysel istatistik metotlar. Köyhizmetleri genel müd. yayinlari. Genel Yayin No: 121. Teknik Yayin No: 56. Ankara.

Zaffaroni, E. and Schneiter, A.A., 1991. Sunflower Production as Influenced by Plant Type, Plant Population, and row Arrangement. Agronomy Journal 83: 113-118. 